\title{
Dependence of Coercivity and Barkhausen Noise Signal on Martensitic Stainless Steel with and without Quench
}

\author{
Hiroaki KIKUCHI $^{\mathrm{a}, 1}$, Kohei SUGAI ${ }^{\mathrm{a}}$, Takeshi MURAKAMI ${ }^{\mathrm{a}}$ \\ and Keiichi MATSUMURA ${ }^{\mathrm{b}}$ \\ ${ }^{a}$ Faculty of Science and Engineering, Iwate University, 4-3-5 Ueda, Morioka, Iwate, \\ 020-8551, Japan \\ ${ }^{\mathrm{b}}$ INFITECHM Co., Ltd., Yokohama, Japan
}

\begin{abstract}
We evaluated hysteresis curves and Barkhausen noise properties of the martensitic stainless steel with and without quench, and then investigated the relations between magnetic parameters and hardness based on microstructure changes. The quench introduced to reduce grain size, which relates to an increment of coercivity and Vickers hardness, a decrease in Barkhausen signal. The magnetic property changes reflect the changes in microstructures and mechanical property appeared on the quenched specimen. The obtained results contribute to aim to develop a magnetic nondestructive evaluation of residual stress appeared on the ferromagnetic steel combined with and without quench.
\end{abstract}

Keywords. Hysteresis curve, Barkhausen noise, coercivity, quench, residual stress

\section{Introduction}

The demands of nondestructive sensing technologies for monitoring degradation and health of infrastructures in power generation plants strongly advance in recent year. The material characterization or nondestructive testing using magnetic measurements such as hysteresis loop [1-6] and Barkhausen noise (MBN) [7-12] have been proposed and it is expected to be a candidate contributes to technologies with rapid diagnosis and low-cost inspection. The steel of turbine components used for a thermal power generation plant is typically quenched to enforce its mechanical performance. On the other hand, quenching induces residual stress into the materials, and also the residual stress is formed during long term operating plants, which sometimes has potential to lead the turbine components to a failure. Therefore, quantitative assessment of the residual stress is important. At present, X-ray diffraction is often used for such request, however, the requirement of pretreatment, time-consuming for measurement, and difficulty of in-situ measurement are disadvantages of the technique. The magnetic measurement may be expected to be an alternative method of X-ray diffraction. Therefore, in this study, as a first step, we investigated the magnetic properties and microstructures of martensitic stainless steel used as turbine component steel for thermal power generation plants when the material includes both non-quenched and

\footnotetext{
${ }^{1}$ Corresponding Author, Hiroaki Kikuchi, Faculty of Science and Engineering, Iwate University, Iwate 020-8550, Japan; E-mail: hkiku@iwate-u.ac.jp.
} 


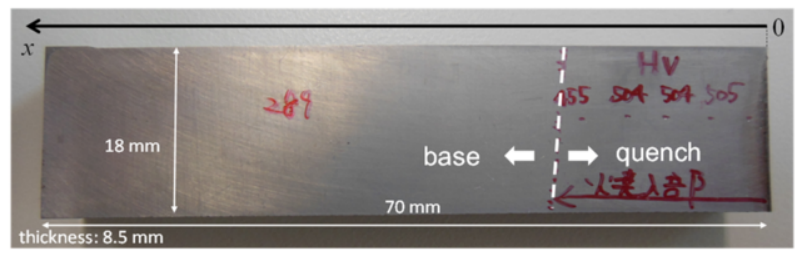

Figure 1 Dimensions and information about specimen used.
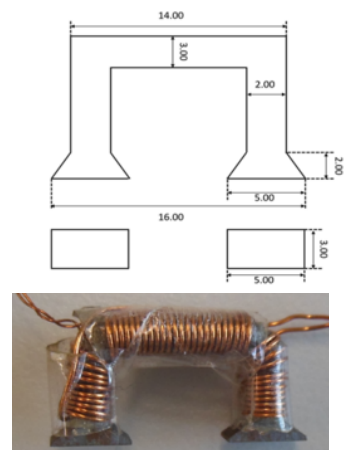

(a) Yoke1
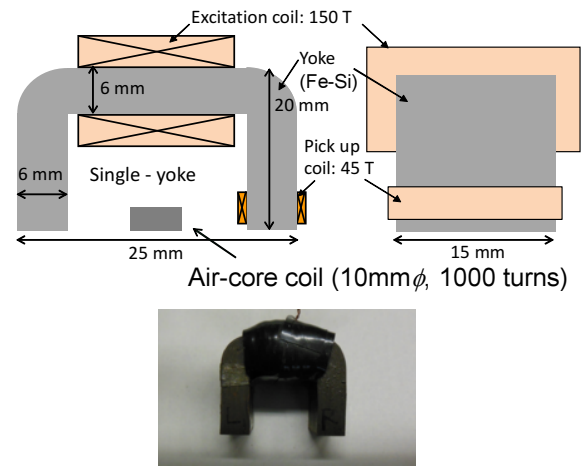

(b) Yoke2

Figure 2 Dimensions and configurations of magnetic yokes. (a) small yoke having 46turns for magnetization and 86turns for pick-up (Yoke1). (b) middle yoke having 150turns for magnetization and 45 turns for pick-up (Yoke2).

quenched area. Although our final goal is to evaluate residual stress quantitatively using magnetic measurement, the magnetic properties are very sensitive to not only stress but also microstructure changes. The main purpose of this study, we discuss magnetic characteristics attributed to microstructures changes due to quenching.

\section{Experimental procedure}

The martensitic stainless steel SUS420J (chemical composition: C 0.17-0.22, Si 0.10$0.50, \mathrm{Mn} 0.30-0.80, \mathrm{P}<0.030, \mathrm{~S}<0.020$, Cr 13.0-14.0, Ni 0.30-0.80, wt.\%) was used for the experiments. The specimen size is $70 \mathrm{~mm} \times 18 \mathrm{~mm} \times 8.5 \mathrm{~mm}$. The specimen was quenched over the area from one of edge to $20 \mathrm{~mm}$ in length direction (See. Figure 1). The Vickers hardness was about $450-500$ in quenched area and was 290 in nonquenched (base) area. We prepared two kinds of yoke in this study: small one (Yoke1) made of pure iron and middle (Yoke2) made of Fe-Si steel. Figure 2 shows the details about dimensions and configurations of both yokes. Since Yoke1 is smaller than Yoke2, its spatial resolution is superior to that of Yoke2. On the other hand, Yoke2 can supply much magnetic flux to the specimen. To investigate these effects, two yokes are used here. The hysteresis curves and MBN properties were measured using magnetic yokes and a pick-up or an air-core coil, respectively. The hysteresis curves were measured using both yokes and MBN signal was measured by Yoke2 only. A triangular current $I$ of 0.1 or $1 \mathrm{~Hz}$ with an amplitude of $1 \mathrm{~A}$ is applied to an excitation coil winding around a yoke for magnetizing a specimen, and an induced voltage at the pick-up coil wounded the yoke leg or the air-core coil located at the surface of the specimen was measured. The output at the pick-up coil is used for calculation of flux 


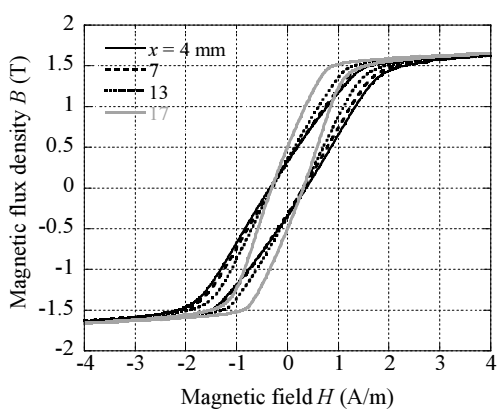

(a) quenched and transition area

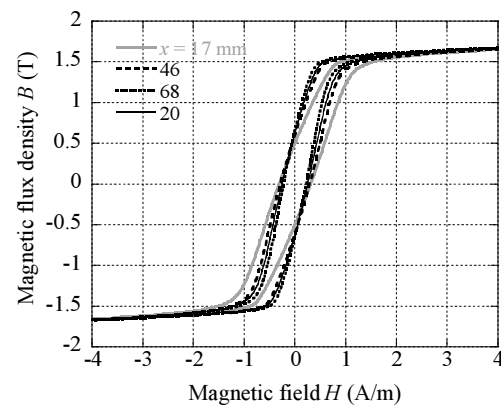

(b) base and transition area

Figure 3. Hysteresis curves measured by small yoke (Yoke1).

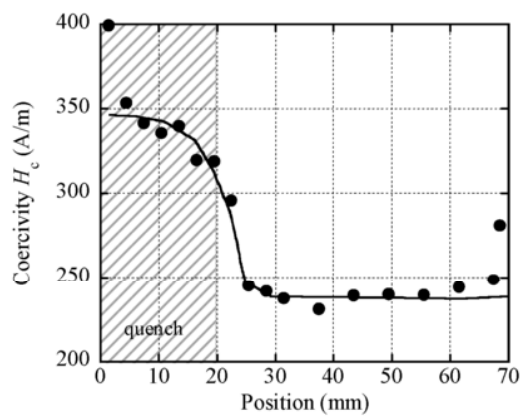

(a) Coercivity

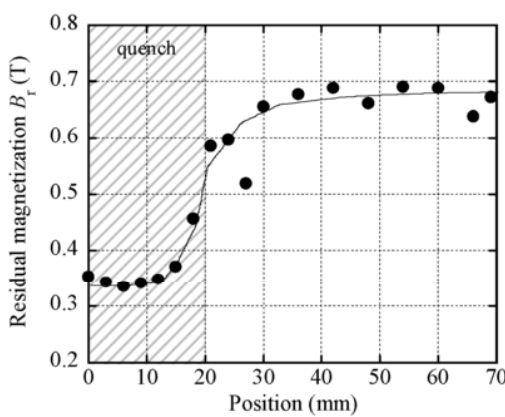

(b) Remanence

Figure 4. Dependence of coercivity and remanence on measurement point.

density $B$ and the output at the air-core coil was amplified by 1000 times, filtered (100 $-200 \mathrm{kHz}$ ) and then captured by a PC as MBN signal. The root mean square value of a half cycle of the output at air-core coil was evaluated as the RMS voltage as following.

$$
R M S=\sqrt{\frac{1}{T} \int_{0}^{T} V_{o}^{2} d t}
$$

where $V_{o}$ is the detected signal after amplified and filtered, and $T$ is a half period of magnetizing field. Based on the results of MBN signal, MBN profile in which the moving averaged rms voltage, $V_{\text {rms }}$ [13] are plotted against the applied current was obtained at each measurement point. As shown in Figure 1, the quenched edge is defined as $x=0$ and length direction of the specimen is $x$-direction. Then the yoke moves along the $x$-direction and hysteresis curves and MBN signal were measured. The sensor positions are defined as the edge of the yoke leg for hysteresis measurement, and as the center of the air-core coil for MBN measurements.

\section{Experiment results}

\subsection{Changes in magnetic parameters on hysteresis curves}

Figure 3 shows the hysteresis curves measured by the small yoke (Yoke 1). The applied magnetic field was parallel to the width direction of the specimen. The curves in the 


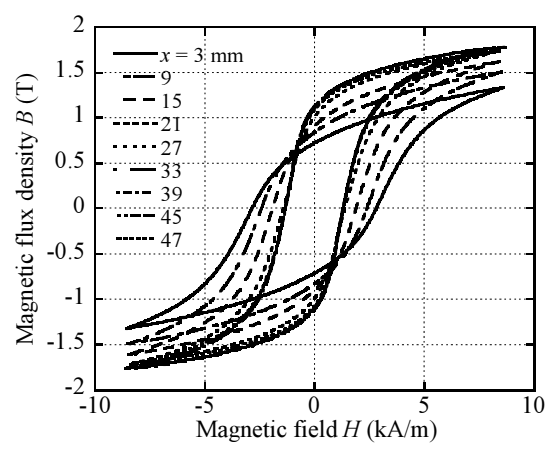

(a) Hysteresis curves

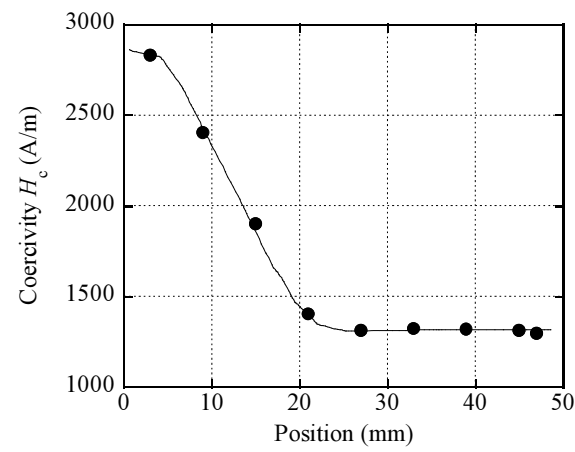

(b) Coercivity vs. position

Figure 5. Hysteresis curves measured by middle yoke (Yoke2) (a) and dependence coercivity on measurement point.

quenched area $(x=4-17 \mathrm{~mm})$ has a small slope and the slope gradually increases with shifting measuring point to the base area. The coercivity and the residual magnetization were obtained from the hysteresis curves shown in Figure 3, and they are plotted against a measuring position. The coercivity shows large value in the quenched area, and has a small value in the base area. In the transition area between quench and nonquench, the coercivity rapidly decreases when measurement point moves from the quenched area to the base. On the other hand, the remanence increases with moving measurement point from quenched area to the base area. We also confirmed that the magnetic parameters correlate with Vickers hardness; the coercivity and the hardness are hard magnetically and mechanically, in quenched area respectively, and they become softer with shifting to the base area.

Figure 5 (a) shows the hysteresis curves measured by the middle yoke (Yoke2). Though the changes in the profile are qualitatively same as the case of Yoke1, we can see clearer changes on the hysteresis curves. The curve becomes swollen with a shifting measurement point from the base to the quenched area. Figure 5 (b) plots the coercivity obtained from the curves in Figure 5 (a) against the measurement point. Though the tendency of change is same as the results of Yoke1, the changing ratio becomes milder; the coercivity drops suddenly at the boundary between quenched and base area in case of Yoke1. This depends on the size of the yoke. Since Yokel is smaller, thus its spatial resolution is higher than that of Yoke2. However, its volume is smaller, which is attributed to less capability supplying magnetic flux to specimen. Indeed, we can understand in Figure 3 that the magnetic yoke is magnetically saturated.

\subsection{MBN profiles}

Figure 6 (a) shows the MBN profiles and Figure 6 (b) shows the position dependence of RMS voltage. The profile shows the results with half cycle, from negative to positive field. Opposite profile, from positive to negative, and the profile in Figure 6 (a) are symmetric with respect to the voltage-axis. The peak height is nearly $100 \mathrm{mV}$ at $I=$ $0.2 \mathrm{~A}$ in the quenched area while the peak height is above $500 \mathrm{mV}$ at $I=0.05 \mathrm{~A}$ on the base area. The profiles show a little bit complicated behavior in the transition area. For example, at $x=6 \mathrm{~mm}$, the profile shows another peak around $I=-0.1 \mathrm{~A}$, and peak position at $0.2 \mathrm{~A}$ in the quenched area shifts to the lower applied current, and its height becomes higher with moving measurement point to the base area. The latter peak may 


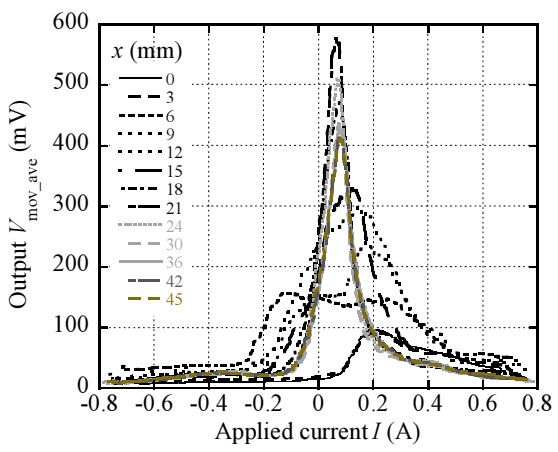

(a) MBN profile

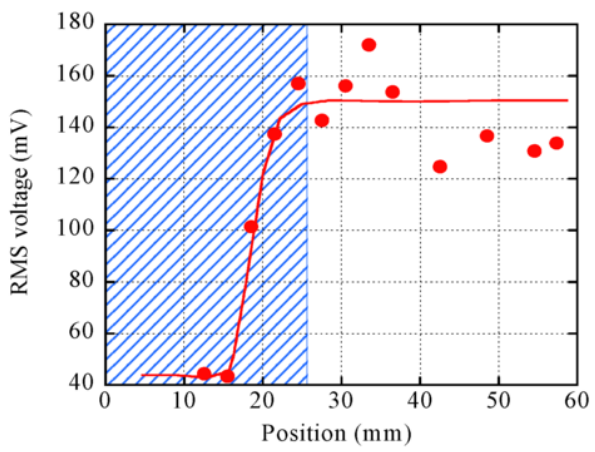

(b) RMS voltage vs. position

Figure 6. MBN profiles measured at different point (a) and position dependence of RMS voltage (b).

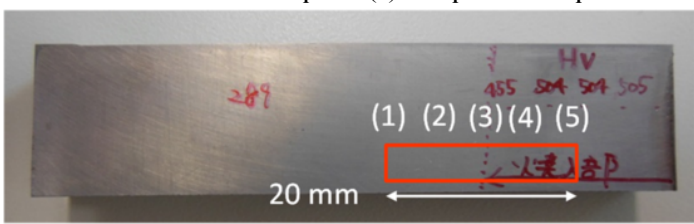

Figure 7. Observation area for EBSD.

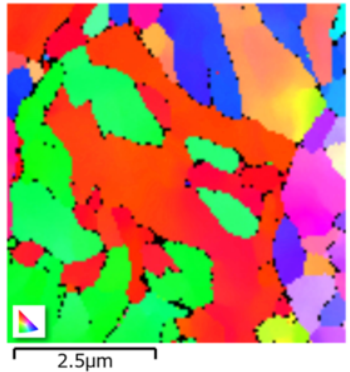

(a) (1)

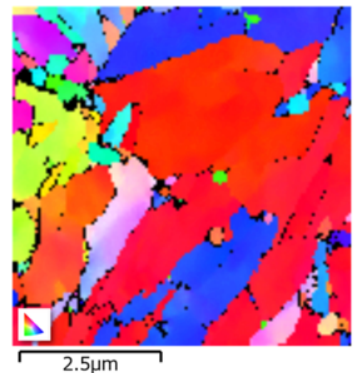

(b) (3)

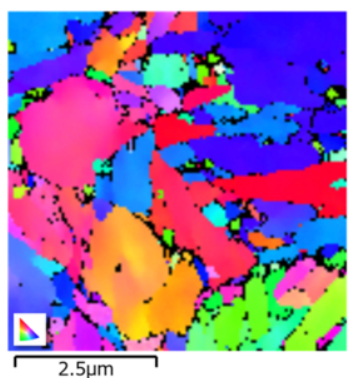

(c) (5)

Figure 8. Orientation map obtained by EBSD observation.

be related to the microstructures change due to quenching, while the former may have relation with another factor; one possibility is due to residual stress. RMS voltage in quenched area shows low values and it increases at the base area.

\section{Discussion}

The obtained results in the previous section indicated that the pinning site for domain wall in the quenched area increases. To investigate the changes in microstructures, microstructure observations using an electron backscatter diffraction (EBSD) have done. The specimen for EBSD was cut from the specimen used for the magnetic measurement. As shown in Figure 7 (a), part (1) and (2) is the base area, (4) and (5) is the quenched area, and (3) is a transition area. Figure 8 shows the orientation map obtained from the EBSD observations. We can see that the grain size in the quenched area becomes fine compared with the base area, which means quench has an effect to reduce grains size, that is, increases grain boundaries.

The magnetic properties and MBN signal originate from the interaction between domain wall and pinning site for domain wall. The grain boundary acts as the 
pinning site for domain wall. Since quenching makes grain small, grain boundary increases, which is consistent with that pinning for domain wall increases. Thus, the coercivity increases and MBN signal decrease in quenched area. The grain boundary also acts as a pinning site for dislocations, which contributes to increases hardness.

\section{Conclusions.}

We evaluated magnetic parameters of martensitic stainless steel with and without quench. The grains become fine due to quench, which introduces the increase in coercivity and the decrease in RMS voltage of MBN. Vickers hardness becomes larger with quench and it has correlations with magnetic parameters. Magnetic properties in a transition area between bases and quench may reflect microstructures change and stress distribution. Though the specimen used here includes residual stress partially, microstructure changes about grain size strongly affect the magnetic properties of hysteresis and RMS voltage. On the other hand, residual stress may be evaluated using analysis based on MBN profiles.

\section{References}

[1] H. Kronmüller, Magnetic Techniques for the Study of Dislocations in Ferromagnetic Materials, International Journal of Nondestructive Testing 3 (1972), 315-350.

[2] D. C. Jiles, Introduction to Magnetism and Magnetic Materials, London UK, Chapman \& Hail (1991).

[3] J. Šternberk, E. Kratochvílová, A. Gemperle, V. Faja, and V. Walder, Dependence of characteristics of hysteresis loops on dislocation densities for low-alloy Cr-Mo steel, Czechoslovak Journal of Physics B 35 (1985), 1259-1266.

[4] A. Martínez-de-Guerenu, K. Gurruchaga, and F. Arizti, Nondestructive characterization of recovery and recrystallization in cold rolled low carbon steel by magnetic hysteresis loops, Journal of Magnetism and Magnetic Materials 316 (2007), e842-e845.

[5] S. Takahashi, J. Echigoya, and Z. Motoki, Magnetization curves of plastically deformed Fe metals and alloys, Journal of Applied Physics 87 (2003), 805-813.

[6] H. Kikuchi, Relationship between magnetic properties and hardness and its effect on recovery and recrystallization in cold rolled steel, IEEE Transactions on Magnetics 51 (2015), article \# 2004804.

[7] J. Degauque, B. Astie, J. L. Porteseil, and R. Vergne, Influence of the grain size on the magnetic and magnetomechanical properties of highpurity iron, Journal of Magnetism and Magnetic Materials 26 (1982), 261-263.

[8] C. Gatelier-Rothea, J. Chicois, R Fougeres, P. Fleischmann, Characterization of pure iron and (130 p.p.m.) carbon-iron binary alloy by Barkhausen noise measurements: study of the influence of stress and microstructure, Acta materialia 46 (1998), 4873-4882.

[9] C. G. Stefanita, D. L. Atherton and L. Clapham, Plastic versus Elastic Deformation Effects on Magnetic Barkhausen Noise in Steel, Acta materialia 48 (2000), 3545-3551.

[10] J. Anglada-Rivera, L. R. Padovese, and J. Capó-Sánchez, Magnetic Barkhausen noise and hysteresis loop in commercial carbon steel: Influence of applied tensile stress and grain size, Journal of Magnetism and Magnetic Material 231 (2001), 299-306.

[11] A. Martínez-de-Guerenu, F. Arizti, M. Díaz-Fuentes, and I. Gutiérrez, Recovery during annealing in a cold rolled low carbon steel. Part I: Kinetics and microstructural characterization, Acta materialia 52 (2004), 3657-3664.

[12] X. Kleber, A. Vincent, On the role of residual internal stresses and dislocations on Barkhausen noise in plastically deformed steel, NDT \& E International 37 (2004), 439-445.

[13] H. Kikuchi, K. Ara, Y. Kamada, and S. Kobayashi, Effect of Microstructure Changes on Barkhausen Noise Properties and Hysteresis Loop in Cold-rolled Low Carbon Steel, IEEE Transactions on Magnetics 45 (2009), 2744-2747. 